\title{
Rakotch type contractive maps, square roots and uniform convexity
}

\section{Antonio Jiménez-Melado*}

\begin{abstract}
In this paper we introduce a family of weakly contractive maps on the space $B(S)$ of bounded real valued functions and use it to show that a fundamental step in the proof of the well-known Stone-Weierstrass approximation theorem can be achieved via Rakotch's fixed point theorem for weakly contractive maps. With the same technique, we obtain Zemanek's theorem on the existence of square roots in certain Banach subalgebras of $B(S)$, and, finally, in the context of abstract Banach algebras, we exhibit some relationship between weakly contractive maps on the closed unit ball and the geometry of the spheres.
\end{abstract}

MSC: 47H09; 46J10; 46B20

Keywords: weakly contractive; Rakotch type contraction; Stone-Weierstrass; Banach algebras; square root

\section{Introduction and preliminary results on weakly contractive maps}

If $(X, d)$ is a metric space and $D$ is a nonempty subset of $X$, then we say that $T: D \rightarrow X$ is contractive if there exists $\alpha \in[0,1)$ such that

$$
d(T(x), T(y)) \leq \alpha d(x, y)
$$

for all $x, y \in X$. The famous Banach-Caccioppoli theorem [1,2] asserts that if $(X, d)$ is complete and $T: X \rightarrow X$ is contractive, then $T$ has a unique fixed point $x^{*} \in X$ and, for any $x_{0} \in X$, the sequence $\left\{T^{n}\left(x_{0}\right)\right\}$ converges to $x^{*}$.

After this theorem, some authors tried to generalize it keeping the conclusions on the existence and uniqueness under more general hypotheses, and in many cases the maps satisfying the new conditions have been named weakly contractive. In this section we will be concerned with three of these conditions, due to Rakotch [3], Krasnosel'skii et al. [4] and Dugundji and Granas [5], which will be shown to be equivalent formulations of the same concept. There are some other conditions which are worth mentioning; for instance, the one introduced by Geraghty [6], which is still object of generalizations (see, for instance, Karapinar [7]), but they are not the subject of study in this paper.

As far as we know, the first result in this direction was given by Rakotch [3] in 1961, who replaced the constant $\alpha$ by a function $\alpha=\alpha(d(x, y))$, allowing it to approach 1 exceptionally: we say that $T: D \subset X \rightarrow X$ is weakly contractive in the sense of Rakotch if for every $x, y \in$

(c) 2015 Jiménez-Melado. This article is distributed under the terms of the Creative Commons Attribution 4.0 International License (http://creativecommons.org/licenses/by/4.0/), which permits unrestricted use, distribution, and reproduction in any medium, provided you give appropriate credit to the original author(s) and the source, provide a link to the Creative Commons license, and indicate if changes were made. 
$D$, we have

$$
d(T(x), T(y)) \leq \alpha(d(x, y)) d(x, y)
$$

where $\alpha:[0, \infty) \rightarrow[0,1]$ is a map that is compactly less than 1 , that is $\alpha$ is a map such that, for all $0<a \leq b$,

$$
\sup \{\alpha(t): a \leq t \leq b\}<1 .
$$

In a subsequent work, Krasnosel'skii et al. (see [4], p.51) proved that the function $\alpha=$ $\alpha(d(x, y))$ that appears in Rakotch's theorem could be replaced by a more general function $\alpha=\alpha(x, y)$ satisfying the property of being compactly less than 1 , that is, a function $\alpha$ : $D \times D \rightarrow[0,1]$ such that, for every $x, y \in D$,

$$
d(T(x), T(y)) \leq \alpha(x, y) d(x, y)
$$

and, for every $a, b \in \mathbb{R}$ with $0<a \leq b$,

$$
\Theta_{\alpha}(a, b)=\sup \{\alpha(x, y): a \leq d(x, y) \leq b\}<1
$$

Another condition, which looks different from (K) and that can substitute (1) in BanachCaccioppoli's theorem, was introduced in 1978 by Dugundji and Granas [5] and reads as follows: there exists $\gamma: D \times D \rightarrow[0, \infty)$ such that

$$
\inf \{\gamma(x, y): a \leq d(x, y) \leq b\}>0
$$

for every $0<a \leq b$ and such that

$$
d(T(x), T(y)) \leq d(x, y)-\gamma(x, y)
$$

for all $x, y \in D$. In the same paper, Dugundji and Granas proved that condition (D-G) is equivalent to condition (K) and, obviously, $(\mathrm{R})$ implies $(\mathrm{K})$; hence, the three conditions would be equivalent if the implication $(K) \Longrightarrow(R)$ were true. Next, we shall show that this is the case, that is, we shall prove that the three conditions are equivalent. We state this as a theorem.

Theorem 1 Let $(X, d)$ be a metric space, and $D$ a nonempty subset of $X$. Then, for any map $T: D \rightarrow X$, conditions $(\mathrm{R}),(\mathrm{K})$ and $(\mathrm{D}-\mathrm{G})$ are equivalent.

Proof As we have previously noticed, we only need to show that (K) implies (R). For that, start supposing that a map $T: D \rightarrow X$ satisfies condition (K), which means the existence of a map $\alpha: D \times D \rightarrow[0,1]$ with $\alpha$ compactly less than 1 and satisfying inequality (K). Define $\bar{\alpha}:[0, \infty) \rightarrow[0,1]$ as

$$
\bar{\alpha}(t)=\sup \{\alpha(x, y): x, y \in D \text { and } d(x, y)=t\}
$$


if there exist $x, y \in D$ with $d(x, y)=t$ and $\bar{\alpha}(t)=\frac{1}{2}$ otherwise. Then, $\bar{\alpha}$ is compactly less than 1 because, for $0<a \leq t \leq b$ and $x, y \in D$ with $d(x, y)=t$, we have $\alpha(x, y) \leq \Theta_{\alpha}(a, b)$, from which $\bar{\alpha}(t) \leq \Theta_{\alpha}(a, b)$, and, consequently, for every $a, b \in \mathbb{R}$ with $0<a \leq b$,

$$
\sup \{\bar{\alpha}(t): a \leq t \leq b\} \leq \max \left\{\frac{1}{2}, \Theta_{\alpha}(a, b)\right\}<1
$$

Observe also that inequality (R) is satisfied with the function $\bar{\alpha}$ because for any $x, y \in D$, we have $\alpha(x, y) \leq \bar{\alpha}(d(x, y))$, and $T$ satisfies condition (K) with the function $\alpha$. Hence, $T$ satisfies condition (R).

The previous result allows us to say that $T: D \subset X \rightarrow X$ is weakly contractive if it satisfies some (hence, all) of conditions (R), (K) or (D-G). Rakotch's theorem [3] states that if $(X, d)$ is a complete metric space and $T: X \rightarrow X$ is weakly contractive, then $T$ has a unique fixed point in $X$, say $x^{*}$, and for each $x_{0} \in X$, the sequence of iterates $\left\{T^{n}\left(x_{0}\right)\right\}$ converges to $x^{*}$. Also, we can see that this theorem is a strict generalization of Banach-Caccioppoli's theorem just considering the space $X=[0,1]$ with the usual metric $d(x, y)=|x-y|$ and the map $T: X \rightarrow X$ given as $T(x)=\sin (x)$, which is weakly contractive but not contractive.

In Section 2 we introduce a family of weakly contractive maps on the space $B(S)$ of bounded real functions and show that Rakotch's theorem can be used to prove a result of Zemanek [8] about the existence of square roots in some Banach subalgebras of $B(S)$, a result which is a fundamental step in the proof of the well-known Stone-Weierstrass approximation theorem [9]. The use of Rakotch's theorem in the proof of this theorem is also interesting because, for weakly contractive maps, it can be given a rate of convergence of the sequence of iterates (see [10]).

In Section 3 we show that there are some connections between weakly contractive maps, square roots in abstract algebras and the geometry of the unit ball.

\section{Weakly contractive maps and square roots in $B(S)$}

As we mentioned in the introduction, there are simple examples of maps that are weakly contractive and not contractive. In this section we provide a new example of this type, which is interesting because it existed before Rakotch's theorem and appears in some proofs of the famous Stone-Weierstrass approximation theorem [9]. The aforementioned example is the map $T$ defined on certain subset of the space $\mathcal{C}([0,1])$ by $T(f)(x)=\frac{1}{2}[1-$ $x+f^{2}(x)$ ], which is used in [11] to prove that the function $\varphi(x)=\sqrt{x}$ can be uniformly approximated by polynomials (using Dini's test for uniform convergence). In fact, a slight modification of this example will allow us to obtain the result of Zemanek [8] about the existence of square roots in certain Banach algebras. In the first place, we recall some notation and results that are necessary to understand the context which we shall work at.

If $S$ is any nonempty set, then $B(S)$ denotes the set of all bounded functions $f: S \rightarrow \mathbb{R}$, which is known to be a Banach algebra with the pointwise multiplication and the norm $\|$. $\|_{\infty}$ (see, for instance, [11]). We also use the notation $B^{+}(S)=\{f \in B(S): f(x) \geq 0$ for all $x \in$ $S\}$ and, for a nonempty subset $J \subset \mathbb{R}, B(S, J)=\{f \in B(S): f(x) \in J$ for all $x \in S\}$.

If $S$ is a compact topological space, then it is also known that the space of continuous real functions $\mathcal{C}(S)$ with its usual norm $\|\cdot\|_{\infty}$ is a Banach subalgebra of $B(S)$. Notice that, by elementary rules of calculus, the subalgebra $\mathcal{C}(S)$ has the following property: for any $f \in$ $B^{+}(S) \cap \mathcal{C}(S)$, we have $\sqrt{f} \in \mathcal{C}(S)$. The question of whether this property could be extended 
to abstract subalgebras of $B(S)$ was answered by Zemanek [8] in 1977 by showing that the aforementioned property is satisfied by those subalgebras $\mathcal{A}$ of $B(S)$ that are complete and contain the constant functions. We obtain this result in Theorem 2 by using Rakotch's theorem, and this will provide us with an extra information about how to obtain $\sqrt{f}$ as a uniform limit of functions of $\mathcal{A}$.

As a first step, in Lemma 1 we introduce a family of weakly contractive maps that will be used in Theorem 2.

Lemma 1 Let $G: S \times J \rightarrow \mathbb{R}$ be a bounded map, where $S$ and $J \subset \mathbb{R}$ are nonempty sets, suppose that $D \subset B(S, J)$ is nonempty and define $T: D \rightarrow B(S)$ as $T(f)(x)=G(x, f(x))$. Then, if $D$ contains the constant functions of $B(S, J)$, then we have that

(a) $T$ is weakly contractive on $D$ if, and only if, there exists a function $\alpha: J \times J \rightarrow[0,1]$ compactly less than 1 such that

$$
|G(x, u)-G(x, v)| \leq \alpha(u, v)|u-v|
$$

for every $u, v \in J$ and every $x \in S$;

(b) $T$ is contractive if, and only if, there exists $\alpha \in[0,1)$ such that

$$
|G(x, u)-G(x, v)| \leq \alpha|u-v|
$$

for every $u, v \in J$ and every $x \in S$.

Proof We shall only prove part (a), and the proof of part (b) is left to the reader. To prove (a), suppose first that there exists $\alpha: J \times J \rightarrow[0,1]$ compactly less than 1 and satisfying (2). Then, we know that for any two real numbers $a$ and $b$ with $0<a \leq b$, we have

$$
\Theta_{\alpha}(a, b)=\sup \{\alpha(u, v): u, v \in J, a \leq|u-v| \leq b\}<1 .
$$

We need to show the existence of a function $\beta: D \times D \rightarrow[0,1]$ with $\beta$ compactly less than 1 and satisfying $\|T(f)-T(g)\|_{\infty} \leq \beta(f, g)\|f-g\|_{\infty}$ for every $f, g \in D$. Indeed, we shall see that the function $\beta$ defined as $\beta(f, f)=0$ and

$$
\beta(f, g)=\max \left\{\frac{1}{2}, \Theta_{\alpha}\left(\frac{1}{2}\|f-g\|_{\infty},\|f-g\|_{\infty}\right)\right\}
$$

for $f \neq g$ satisfies all our requirements. Obviously, $\beta$ takes values in $[0,1]$, and, for all real numbers $0<a \leq b$, we have

$$
\Theta_{\beta}(a, b) \leq \max \left\{\frac{1}{2}, \Theta_{\alpha}\left(\frac{1}{2} a, b\right)\right\}<1,
$$

which shows that $\beta$ is compactly less than 1 . Now, observe that for $f, g \in D$ and $x \in S$, we have

$$
\begin{aligned}
|T(f)(x)-T(g)(x)| & =|G(x, f(x))-G(x, g(x))| \\
& \leq \alpha(f(x), g(x))|f(x)-g(x)|
\end{aligned}
$$


and argue as follows: if $|f(x)-g(x)| \leq \frac{1}{2} \mid f-g \|_{\infty}$, then use that $0 \leq \alpha(f(x), g(x)) \leq 1$ together with (3) to obtain $|T(f)(x)-T(g)(x)| \leq \frac{1}{2}\|f-g\|_{\infty}$; otherwise, that is, if $\frac{1}{2}\|f-g\|_{\infty}<\mid f(x)-$ $g(x) \mid \leq\|f-g\|_{\infty}$, then use that $\alpha(f(x), g(x)) \leq \Theta_{\alpha}\left(\frac{1}{2}\|f-g\|_{\infty},\|f-g\|_{\infty}\right)$ to obtain

$$
|T(f)(x)-T(g)(x)| \leq \Theta_{\alpha}\left(\frac{1}{2}\|f-g\|_{\infty},\|f-g\|_{\infty}\right)\|f-g\|_{\infty} .
$$

Hence, we have proved that $\|T(f)-T(g)\|_{\infty} \leq \beta(f, g)\|f-g\|_{\infty}$.

For the converse, suppose that $T$ is weakly contractive, that is, there exists $\beta: D \times D \rightarrow$ $[0,1]$ compactly less than 1 and satisfying $\|T(f)-T(g)\|_{\infty} \leq \beta(f, g)\|f-g\|_{\infty}$ for all $f, g \in D$, and define $\alpha: J \times J \rightarrow[0,1]$ as $\alpha(u, v)=\beta\left(f_{u}, f_{v}\right)$, where, for each $w \in J, f_{w}: S \rightarrow J$ denotes the constant function $f_{w}(x)=w$. We have to show that $\alpha$ is compactly less than 1 and satisfies (2); for the former, just observe that for any two real numbers $0<a \leq b$, we have

$$
\begin{aligned}
\Theta_{\alpha}(a, b) & =\sup \{\alpha(u, v): u, v \in J, a \leq|u-v| \leq b\} \\
& =\sup \left\{\beta\left(f_{u}, f_{v}\right): u, v \in J, a \leq\left\|f_{u}-f_{v}\right\|_{\infty} \leq b\right\} \\
& \leq \sup \left\{\beta(f, g): f, g \in D, a \leq\|f-g\|_{\infty} \leq b\right\} \\
& =\Theta_{\beta}(a, b)<1 .
\end{aligned}
$$

For the latter, suppose that $x \in S$ and $u, v \in J$ and obtain

$$
\begin{aligned}
|G(x, u)-G(x, v)| & =\left|G\left(x, f_{u}(x)\right)-G\left(x, f_{v}(x)\right)\right| \\
& =\left|T\left(f_{u}\right)(x)-T\left(f_{v}\right)(x)\right| \\
& \leq \beta\left(f_{u}, f_{v}\right) \mid f_{u}-f_{v} \|_{\infty} \\
& =\alpha(u, v)|u-v| .
\end{aligned}
$$

Theorem 2 Let $\mathcal{A}$ be a complete subalgebra of $B(S)$ that contains the constant functions. Then, for each $g^{*} \in B^{+}(S) \cap \mathcal{A}$ with $M=\left\|g^{*}\right\|_{\infty}>0$, we have:

(a) $\sqrt{g^{*}} \in \mathcal{A}$, and

(b) the sequence of functions $\left\{g_{n}\right\}$ given as $g_{0}(x)=0$ and

$$
g_{n}(x)=g_{n-1}(x)+\frac{1}{2 \sqrt{M}}\left[g^{*}(x)-g_{n-1}^{2}(x)\right], \quad n \geq 1,
$$

is a sequence in $\mathcal{A}$ that converges uniformly on $S$ to $\sqrt{g^{*}}$.

Proof Suppose that $g^{*} \in B^{+}(S) \cap \mathcal{A}$ with $M=\left\|g^{*}\right\|_{\infty}>0$. Observe that since $\mathcal{A}$ contains the constant functions, $\sqrt{g^{*}}$ belongs to $A$ if, and only if, the function $f^{*}$ given as $f^{*}(x)=\sqrt{M}-$ $\sqrt{g^{*}(x)}$ is in $\mathcal{A}$. It is also obvious that the sequence $\left\{g_{n}\right\}$ is in $\mathcal{A}$ and converges uniformly to $\sqrt{g^{*}}$ if, and only if, the sequence $\left\{f_{n}\right\}$ defined as $f_{n}=\sqrt{M}-g_{n}$ is in $\mathcal{A}$ and converges uniformly to $f^{*}$.

Hence, we only need prove that $f^{*} \in \mathcal{A}$ and that the sequence $\left\{f_{n}\right\}$ is in $\mathcal{A}$ and converges uniformly to $f^{*}$. For that purpose, observe first that the sequence $\left\{f_{n}\right\}$ can be expressed as $f_{0}(x)=\sqrt{M}$ and, for $n \geq 1$,

$$
f_{n}(x)=\frac{1}{2 \sqrt{M}}\left[M-g^{*}(x)+f_{n-1}^{2}(x)\right] .
$$


Then, consider the set $K=\mathcal{A} \cap B(S,[0, \sqrt{M}])$, define $T: K \rightarrow B(S)$ by

$$
T(f)(x)=\frac{1}{2 \sqrt{M}}\left[M-g^{*}(x)+f^{2}(x)\right]
$$

and observe that $f^{*}$ is the unique fixed point for $T$ in the set $B(S,[0, \sqrt{M}])$. Observe also that $\left\{f_{n}\right\}$ is the sequence of iterates for $T$ with starting point $f_{0}(x)=\sqrt{M}$, so that the proof of (a) and (b) will follow from Rakotch's theorem [3] once we see that $T$ is weakly contractive and that $K$ is closed and invariant under $T$.

It is easily seen that $K$ is closed using that $\mathcal{A}$ is closed and that the uniform convergence implies pointwise convergence, and it is easily shown that $T(K) \subset K$ using both that $\mathcal{A}$ is a subalgebra that contains the constant functions and that $0 \leq T(f)(x) \leq \sqrt{M}$ whenever $0 \leq f(x) \leq \sqrt{M}$. Finally, to check that $T$ is weakly contractive, we shall use part (a) of Lemma 1. For that, observe that $T$ can be written as $T(f)(x)=G(x, f(x))$, where $J=[0, \sqrt{M}]$, $D=K$ and $G: S \times J \rightarrow \mathbb{R}$ is defined by

$$
G(x, u)=\frac{1}{2 \sqrt{M}}\left[M-g^{*}(x)+u^{2}\right] .
$$

Obviously, $G$ is bounded, $D$ contains the constant functions of $B(S, J)$, and, for $x \in S$ and $u, v \in J$, we have

$$
\begin{aligned}
|G(x, u)-G(x, v)| & =\frac{1}{2 \sqrt{M}}\left|u^{2}-v^{2}\right| \\
& \leq \alpha(u, v)|u-v|,
\end{aligned}
$$

where $\alpha: J \times J \rightarrow[0,1]$ is the map given as $\alpha(u, v)=\frac{u+v}{2 \sqrt{M}}$.

To see that $\alpha$ is compactly less than 1 , just observe that, for $0<a \leq b$,

$$
\begin{aligned}
\Theta_{\alpha}(a, b) & =\sup \left\{\frac{u+v}{2 \sqrt{M}}: u, v \in J, a \leq|u-v| \leq b\right\} \\
& \leq 1-\frac{a}{2 \sqrt{M}} .
\end{aligned}
$$

Hence, by Rakotch's theorem [3], $T$ has a unique fixed point in $K$, and, for any $h \in K$, the sequence of iterates $\left\{T^{n}(h)\right\}$ converges uniformly on $S$ to this fixed point. Since $K \subset B(S, J)$ and $f^{*}$ is the unique fixed point for $T$ in $B(S, J)$, we conclude that $f^{*} \in K$. Also, starting with $h=f_{0} \in K$, the sequence $\left\{T^{n}(h)\right\}$ is just $\left\{f_{n}\right\}$, and so it converges uniformly to $f^{*}$.

Notice that Theorem 2 generalizes the following well-known result, which our operator $T$ is inspired on.

Corollary 1 (Dieudonné $[11]$ ) The sequence $\left\{g_{n}\right\}$ in $\mathcal{C}([0,1])$ given as $g_{0}(x)=0$ and

$$
g_{n}(x)=g_{n-1}(x)+\frac{1}{2}\left[x-g_{n-1}^{2}(x)\right], \quad n \geq 1,
$$

is a sequence of polynomials that converges uniformly to the function $\varphi(x)=\sqrt{x}$ on the interval $[0,1]$. 
The map $T: K \rightarrow B(S)$ appearing in the previous theorem is of the form $T(f)(x)=$ $a(x)+F(f(x))$, where in our case $a(x)=\frac{M-g^{*}(x)}{2 \sqrt{M}}$ and $F(u)=\frac{1}{2 \sqrt{M}} u^{2}$. Applying Lemma 1, we obtained that $T$ is weakly contractive on $K$ if, and only if, $F$ is weakly contractive on the interval $J$, which suggests the following question.

Question 1 Find an easy-to-check characterization of weakly contractive maps on the real line.

\section{Weakly contractive maps and the geometry of spheres in abstract algebras}

As we mentioned before, Zemanek [8] proved that if $\mathcal{A}$ is a complete subalgebra of $B(S)$ that contains the constant functions, then for any $g \in B^{+}(S) \cap \mathcal{A}$, we have $\sqrt{g} \in \mathcal{A}$. In his proof, Zemanek used the Banach-Caccioppoli theorem and was inspired by a previous paper by Bonsall and Stirling [12] about the existence of square roots in abstract algebras. Specifically, the following result was proved in [12]: if $\mathcal{A}$ is a real or complex Banach algebra, then for each $a \in \mathcal{A}$ with $\|a\|<1$, there exists a unique $x \in \mathcal{A}$ such that $\|x\|<1$ and $x=\frac{1}{2}\left(a+x^{2}\right)$. The proof of this fact is easy if we additionally assume that $\mathcal{A}$ is commutative: just consider a positive number $d$ with $\|a\| \leq d<1$ and check that the map $T: \bar{B}(0, d) \rightarrow \bar{B}(0, d)$ given as $T(x)=\frac{1}{2}\left(a+x^{2}\right)$ is contractive.

Hence, it is natural to ask whether Bonsall and Stirling's result is true for $\|a\|=1$. If we wanted to prove that the answer is yes, a possible strategy could be repeating the previous argument with $d=1$, that is, we would need to prove that the map $T: \bar{B}(0,1) \rightarrow \bar{B}(0,1)$ defined by $T(x)=\frac{1}{2}\left(a+x^{2}\right)$ has a fixed point in $\bar{B}(0,1)$. Unfortunately, this map needs not be contractive, as it can be seen by considering $\mathcal{A}=\mathbb{R}$ and $a=1$. More generally, if $\mathcal{A}$ is an abstract algebra containing an element $b$ with $\|b\|=\left\|b^{2}\right\|=1$, then $T$ is not contractive. To see this, consider the sequences $\left\{x_{n}\right\}$ and $\left\{y_{n}\right\}$ in $\bar{B}(0,1)$ given as $x_{n}=b$ and $y_{n}=\left(1-\frac{1}{n}\right) b$, $n \geq 1$, and check that

$$
\left\|T\left(x_{n}\right)-T\left(y_{n}\right)\right\|=\left(1-\frac{1}{2 n}\right)\left\|x_{n}-y_{n}\right\|, \quad n \geq 1 .
$$

In view of the above, it is natural to ask whether $T$ is weakly contractive. In the next theorem we show that the answer is yes if $\mathcal{A}$ is commutative and the norm on $\mathcal{A}$ is a uniformly convex norm (that is, for each $\varepsilon \in(0,2]$, there exists $\delta>0$ such that for any $x, y \in \bar{B}(0,1)$ with $\|x-y\| \geq \varepsilon$, we have $\left.\left\|\frac{1}{2}(x+y)\right\| \leq 1-\delta\right)$. Before that, we shall show that the notion of a function compactly less than 1 can be used to characterize the uniform convexity of a Banach space.

Lemma 2 A Banach space $(X,\|\cdot\|)$ is uniformly convex if, and only if, the map $\alpha: \bar{B}(0,1) \times$ $\bar{B}(0,1) \rightarrow[0,1]$ given as $\alpha(x, y)=\left\|\frac{x+y}{2}\right\|$ is compactly less than 1 .

Proof We start supposing that $(X,\|\cdot\|)$ is uniformly convex and prove that $\alpha$ is compactly less than 1 . Indeed, for $0<a \leq b$, we have

$$
\begin{aligned}
\Theta_{\alpha}(a, b) & =\sup \{\alpha(x, y): x, y \in \bar{B}(0,1), a \leq\|x-y\| \leq b\} \\
& \leq \sup \left\{\left\|\frac{x+y}{2}\right\|: x, y \in \bar{B}(0,1), a \leq\|x-y\|\right\} \leq 1-\delta(a)<1 .
\end{aligned}
$$


For the converse, assume that $\alpha$ is compactly less than 1 and prove that $(X,\|\cdot\|)$ is uniformly convex. Hence, let $\varepsilon \in(0,2]$ and choose $\delta=1-\Theta_{\alpha}(\varepsilon, 2)$. Then we have $\delta>0$ and, for every $x, y \in \bar{B}(0,1)$ with $\|x-y\| \geq \varepsilon$,

$$
\left\|\frac{x+y}{2}\right\|=\alpha(x, y) \leq \Theta_{\alpha}(\varepsilon, 2)=1-\delta .
$$

Theorem 3 If $\mathcal{A}$ is a commutative Banach algebra and the norm on $\mathcal{A}$ is a uniformly convex norm, then for each $a \in \bar{B}(0,1)$, the map $T: \bar{B}(0,1) \rightarrow \bar{B}(0,1)$ given as $T(x)=\frac{1}{2}(a+$ $\left.x^{2}\right)$ is weakly contractive.

Proof If $x, y \in \bar{B}(0,1)$, then using the commutativity of $\mathcal{A}$, we obtain

$$
\begin{aligned}
T(x)-T(y) & =\frac{1}{2} x^{2}-\frac{1}{2} y^{2} \\
& =\frac{x+y}{2} \cdot(x-y) .
\end{aligned}
$$

Then, since the norm $\|\cdot\|$ is an algebra norm, we conclude that

$$
\begin{aligned}
\|T(x)-T(y)\| & \leq\left\|\frac{x+y}{2}\right\|\|x-y\| \\
& =\alpha(x, y)\|x-y\|,
\end{aligned}
$$

where $\alpha: \bar{B}(0,1) \times \bar{B}(0,1) \rightarrow[0,1]$ is given as $\alpha(x, y)=\left\|\frac{x+y}{2}\right\|$.

Finally, $\alpha$ is compactly less than 1 by Lemma 2 .

The converse of the theorem is not true, even under the presence of commutativity, as the following example shows.

Example 1 Consider the algebra $\mathcal{A}=\mathcal{C}([0,1])$ with the pointwise multiplication and its usual norm $\|\cdot\|_{\infty}$. Then, $\mathcal{A}$ is a commutative Banach algebra whose norm is not uniformly convex, but, for any $f_{0} \in \mathcal{A}$, the map $T: \bar{B}(0,1) \rightarrow \mathcal{A}$ given as $T(f)=\frac{1}{2}\left(f_{0}+f^{2}\right)$ is weakly contractive.

It is well known that $\mathcal{A}$ is a commutative Banach algebra and also that its norm is not uniformly convex. To see that $T$ is weakly contractive on $\bar{B}(0,1)$, we define $\beta: \bar{B}(0,1) \times$ $\bar{B}(0,1) \rightarrow[0,1]$ by $\beta(f, g)=\max \left\{\frac{1}{2}, 1-\frac{1}{4}\|f-g\|_{\infty}\right\}$ and prove that $\beta$ is compactly less than 1 and also satisfies

$$
\|T(f)-T(g)\|_{\infty} \leq \beta(f, g)\|f-g\|_{\infty}
$$

for every $f, g \in \bar{B}(0,1)$.

First, $\beta$ is compactly less than 1 because for all real numbers $a$ and $b$ with $0<a \leq b$, we have

$$
\begin{aligned}
\Theta_{\beta}(a, b) & =\sup \left\{\beta(f, g): f, g \in \bar{B}(0,1), a \leq\|f-g\|_{\infty} \leq b\right\} \\
& \leq \max \left\{\frac{1}{2}, 1-\frac{a}{4}\right\}<1 .
\end{aligned}
$$


Second, in order to check inequality (5), suppose that $f, g \in \bar{B}(0,1)$ and $x \in[0,1]$ and argue as follows: if $|f(x)-g(x)| \leq \frac{1}{2}\|f-g\|_{\infty}$, then, since $|f(x)+g(x)| \leq 2$, we have

$$
\begin{aligned}
|T(f)(x)-T(g)(x)| & =\frac{1}{2}|f(x)+g(x)| \cdot|f(x)-g(x)| \\
& \leq \frac{1}{2}\|f-g\|_{\infty} .
\end{aligned}
$$

Otherwise, we would have $|f(x)+g(x)| \leq 2-\frac{1}{2}\|f-g\|_{\infty}$, and then

$$
|T(f)(x)-T(g)(x)| \leq\left(1-\frac{1}{4}\|f-g\|_{\infty}\right)|f(x)-g(x)| .
$$

Then, we have that $|T(f)(x)-T(g)(x)| \leq \beta(f, g)\|f-g\|_{\infty}$, and since $x$ is arbitrary in $[0,1]$, (5) is satisfied.

Remark 1 Notice that, although the map $T$ in the example is weakly contractive, the function $\alpha(f, g)=\left\|\frac{1}{2}(f+g)\right\|$ is not compactly less than 1 on $\bar{B}(0,1) \times \bar{B}(0,1)$, as it can be seen either directly or using Lemma 2.

We end this paper with some natural questions related to Theorem 3:

Question 2 Can we remove the commutativity assumption?

Question 3 Can we replace the uniform convexity by a weaker geometrical property?

Question 4 Which consequences (geometrical or not) can we obtain from the fact that the map $T$ is weakly contractive?

Question 5 Can we extend Theorem 3 to the context of Banach spaces (using a different map $T)$ ?

\section{Competing interests}

The author declares that they have no competing interests.

\section{Acknowledgements}

The work was partially supported by the Spanish (Grant MTM2014-52865-P) and regional Andalusian (Grants FQM210 and P06-FQM01504) Governments.

Received: 20 September 2015 Accepted: 25 November 2015 Published online: 08 December 2015

\section{References}

1. Banach, S: Sur les operations dans les ensembles abstraits et leur applications aux équations intégrales. Fundam. Math. 3, 133-181 (1922)

2. Caccioppoli, R: Un teorema generale sull'esistenza di elementi uniti in una transformazione funzionale. Atti Accad. Naz. Lincei (6) 11, 794-799 (1930)

3. Rakotch, E: On $\varepsilon$-contractive mappings. Bull. Res. Counc. Isr., Sect. F 10F, 53-58 (1961)

4. Krasnosel'skii, MA, Vainikko, GM, Zabreiko, PP, Rutitskii, YB, Stetsenko, VY: Approximate Solution of Operator Equations. Wolters-Noordhoff, Groningen (1972) (translated from the Russian by D Louvish)

5. Dugundji, J, Granas, A: Weakly contractive maps and elementary domain invariance theorem. Bull. Soc. Math. Grece 19, 141-151 (1978)

6. Geraghty, M: On contractive mappings. Proc. Am. Math. Soc. 40, 604-608 (1973)

7. Karapinar, E: $\alpha-\Psi$-Geraghty contraction type mappings and some related fixed point results. Filomat $28,37-48$ (2014)

8. Zemanek, J: A simple proof of the Weierstrass-Stone theorem. Comment. Math. Prace Mat. 20, 495-497 (1977/78)

9. Stone, MH: The generalized Weierstrass approximation theorem. Math. Mag. 21, 167-184 (1948) 
10. Ariza-Ruiz, D, Martol Briseid, E, Jiménez-Melado, A, López-Acedo, G: Rate of convergence under weak contractiveness conditions. Fixed Point Theory 14, 11-27 (2013)

11. Dieudonné, J: Foundations of Modern Analysis. Academic Press, New York (1960)

12. Bonsall, FF, Stirling, DSG: Square roots in Banach $*$-algebras. Glasg. Math. J. 13, 74 (1972)

Submit your manuscript to a SpringerOpen ${ }^{\circ}$ journal and benefit from:

- Convenient online submission

Rigorous peer review

- Immediate publication on acceptance

- Open access: articles freely available online

- High visibility within the field

- Retaining the copyright to your article

Submit your next manuscript at $\boldsymbol{s p r i n g e r o p e n . c o m ~}$ 